Supporting Information

\title{
Insights into Electrocatalytic Oxygen Evolution over Hierarchical $\mathrm{FeCo}_{2} \mathrm{~S}_{4}$ Nanospheres
}

Ching-Wen Liao, ${ }^{\dagger, \Delta}$ Sheng-Yu Chen, ${ }^{\dagger}$ Liang-Ching Hsu, ${ }^{\S}$ Chia-Wei Lin, ${ }^{\triangle}$ Jeng-Lung

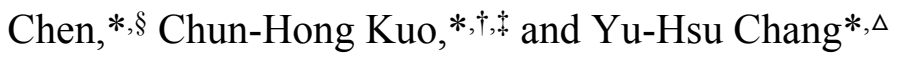

${ }^{\dagger}$ Institute of Chemistry, Academia Sinica, Taipei 115201, Taiwan

${ }^{\triangle}$ Department of Materials and Mineral Resources Engineering, Institute of Mineral

Resources Engineering, National Taipei University of Technology, Taipei 106344, Taiwan

$\S$ National Synchrotron Radiation Research Center, Hsinchu 300092, Taiwan

$\ddagger$ Department of Applied Chemistry, National Yang Ming Chiao Tung University, Hsinchu

300093, Taiwan

\section{Contact information of corresponding authors}

*(J.-L. C.) E-mail: chen.j1@nsrrc.org.tw

*(C.-H. K.) E-mail: chunhong@nycu.edu.tw

*(Y.-H. C.) E-mail: yhchang@ntut.edu.tw 
Number of Pages: 16, Number of Tables: 2, Number of Figures: 11, Number of Scheme: 1

Table S1. Kinetic parameters of Reported $\mathrm{FeCo}_{2} \mathrm{~S}_{4}$ electrocatalysts for Oxygen Evolution

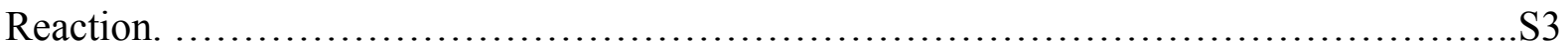

Figure S1. (a, b) SEM images of excavated $\mathrm{FeCo}_{2} \mathrm{O}_{4}$ nanospheres, and (c) its corresponding

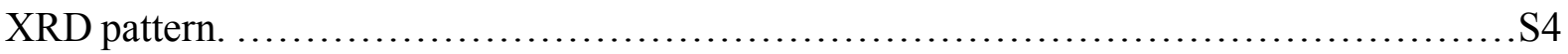

Figure S2. SEM images and STEM-EDS maps of $\mathrm{FeCo}_{2} \mathrm{~S}_{4}$ ENSs made by vulcanization with (a-f) $0.01 \mathrm{M}$, (g-1) $0.05 \mathrm{M}$, (m-r) $0.5 \mathrm{M}$, and (s-x) $1 \mathrm{M} \mathrm{Na}_{2} \mathrm{~S}$.

Figure S3. PXRD patterns of Fe-Co HNSs prepared by vulcanization with $0\left(\mathrm{FeCo}_{2} \mathrm{O}_{4}\right), 0.01$, $0.05,0.1,0.5$, and $1 \mathrm{M} \mathrm{Na}_{2} \mathrm{~S}$. .S6

Figure S4. XPS spectra of $\mathrm{Fe}(2 \mathrm{p}), \mathrm{Co}(2 \mathrm{p}), \mathrm{S}(2 \mathrm{p}), \mathrm{O}(1 \mathrm{~s})$, and their fitting curves for $\mathrm{FeCo}_{2} \mathrm{~S}_{4} / \mathrm{NF}$ made with (a-d) 0.01, (e-h) 0.05, (i-1) 0.5, (m-p) $1 \mathrm{M} \mathrm{Na}_{2} \mathrm{~S}$.

Scheme S1. (a) Schematic illustration for the setup in the H-shaped electrolyzer. (b) Photo of the home-made working electrode. .S8

Figure S5. CVs of (a) $\mathrm{Ni}$ foam, (b) $\mathrm{FeCo}_{2} \mathrm{O}_{4} / \mathrm{NF}$, and (c) $\mathrm{FeCo}_{2} \mathrm{~S}_{4} / \mathrm{NF}$ in $1 \mathrm{M} \mathrm{NaOH}$ at a scan rate of $100 \mathrm{mV} / \mathrm{s}$. (d) The LSV curves of $\mathrm{FeCo}_{2} \mathrm{~S}_{4} / \mathrm{NF}(0.1 \mathrm{M})$ and references.

Figure S6. CV curves at scan rates $(r)$ from 20 to $120 \mathrm{mV} / \mathrm{s}$ and their corresponding plots of $\Delta j$ vs $r$ for $(\mathrm{a}, \mathrm{b}) 0.01 \mathrm{M}-$, (c, d) $0.05 \mathrm{M}-$, (e, f) $0.1 \mathrm{M}-,(\mathrm{g}, \mathrm{h}) 0.5 \mathrm{M}-$, and $(\mathrm{i}, \mathrm{j}) 1.0 \mathrm{M}-\mathrm{FeCo}_{2} \mathrm{~S}_{4} / \mathrm{NF}$ electrodes.

Figure S7. (a) Chronopotentiometry (CP) plot, (b) LSV curve, (c, d) SEM images, and (e) PXRD pattern of $\mathrm{FeCo}_{2} \mathrm{~S}_{4}$ ENSs made with $0.1 \mathrm{M} \mathrm{Na}_{2} \mathrm{~S}$ after 12-hour durability test. The $\mathrm{CP}$ plot was obtained by running scan at a static current density of $50 \mathrm{~mA} \mathrm{~cm}{ }^{-2}$ for 12 hours.

Figure S8. XPS spectra of (a) Fe 2p, (b) Co 2p, (c) S 2p, and (d) O 1s and their fitting curves for $\mathrm{FeCo}_{2} \mathrm{~S}_{4} / \mathrm{NF}\left(0.1 \mathrm{M} \mathrm{Na}_{2} \mathrm{~S}\right)$ after running 12-hour OER.

Figure S9. (a) The scheme and (b) photo of the setup for in-situ XRD and XAS measurements during OER at different potentials.

Figure S10. The $k^{3}$-weighted Fourier-transformed EXAFS spectra of Fe K-edge for the $0.1 \mathrm{M}$ $\mathrm{FeCo}_{2} \mathrm{~S}_{4} \mathrm{HNS}$ obtained in the electrolyte of $1 \mathrm{M} \mathrm{NaOH}$ at different potentials. S14

Figure S11. The $k$ space of Co $K$-edge EXAFS spectra for $0.1 \mathrm{M}-\mathrm{FeCo}_{2} \mathrm{~S}_{4} \mathrm{HNS}$ at different potentials. S15

Table S2. Structure parameters obtained from in-situ Co $K$-edge EXAFS of the $0.1 \mathrm{M}-\mathrm{FeCo}_{2} \mathrm{~S}_{4}$ HNS during OER at different potentials. S16 
Table S1. Kinetic parameters of Reported $\mathrm{FeCo}_{2} \mathrm{~S}_{4}$ electrocatalysts for Oxygen Evolution Reaction.

\begin{tabular}{|c|c|c|c|c|c|c|}
\hline Catalysts & Morphology & Electrolytes & Substrate & $\begin{array}{l}\text { Overpotential (mV) at } \\
\text { sperific entrent density }\end{array}$ & $\begin{array}{l}\text { Tafd slope } \\
\left(\mathrm{m} V \text { dec }^{-1}\right)\end{array}$ & Reference \\
\hline $\mathrm{FeCO}_{2} \mathrm{~S}_{4}$ & Nanosheets & $1 \mathrm{M} \mathrm{NaOH}$ & $N_{1}$ foam & $418 \mathrm{mV} @ 100 \mathrm{mAcm}^{-2}$ & $318 \mathrm{mV} \mathrm{dec}^{-1}$ & This work \\
\hline $\mathrm{FeCl}_{2} \mathrm{~S}_{4}$ & Nanosheet arrays & $1 \mathrm{M} \mathrm{KOH}$ & $N_{1}$ foam & $290 \mathrm{mV} @ 100 \mathrm{mAcm}^{-2}$ & $59 \mathrm{mV} \mathrm{dec}^{-1}$ & $\begin{array}{l}\text { Xiao Peng - ACS Sustainable } \\
\text { Chem. Eng. } 2018,6 \text {, } \\
11724-11733\end{array}$ \\
\hline $\mathrm{FeCl}_{2} \mathrm{~S}_{4}$ & Nanotube arrays & $1 \mathrm{M} \mathrm{KOH}$ & $\begin{array}{l}\text { Carbon } \\
\text { cloths }\end{array}$ & $317 \mathrm{mV} @ 100 \mathrm{mAcm}^{-2}$ & $36 \mathrm{mV} \mathrm{dec}^{-1}$ & $\begin{array}{l}\text { Xuefeng Wang - ACS } \\
\text { Sustainable Chem. Eng. 2019,7, } \\
\text { 19426-19433 }\end{array}$ \\
\hline $\mathrm{FeC}_{0_{2}} \mathrm{~S}_{4}$ & Nanorod arrays & $1 \mathrm{M} \mathrm{KOH}$ & Nifoam & $293 \mathrm{mV} @ 100 \mathrm{mAcm}^{-2}$ & $122.2 \mathrm{mV} \mathrm{dec}^{-1}$ & $\begin{array}{l}\text { Yimin Cui - Catal. Sci. Technol., } \\
2020,10,1292-1298\end{array}$ \\
\hline $\mathrm{FeCO}_{2} \mathrm{~S}_{4}$ & Nanorod arrays & $1 \mathrm{M} \mathrm{KOH}$ & $N_{1}$ foam & $360 \mathrm{mV} @ 75 \mathrm{mAcm}^{-2}$ & $120 \mathrm{mV} \mathrm{dec}^{-1}$ & $\begin{array}{l}\text { Xiaoqiang Du - Int. J. Hydrogen } \\
\text { Energy, 2019, 44, 31902-31915 }\end{array}$ \\
\hline $\begin{array}{l}\mathrm{FeC}_{2} \mathrm{O}_{4} @ \\
\mathrm{FeCO}_{2} \mathrm{~S}_{4}\end{array}$ & Nanoarrays & $1 \mathrm{M} \mathrm{KOH}$ & $N_{1}$ foam & $380.7 \mathrm{mV} @ 50 \mathrm{~mA} \mathrm{~cm}{ }^{-2}$ & $80.3 \mathrm{mV} \mathrm{dec}^{-1}$ & $\begin{array}{l}\text { Xiang Wu - Nano Energy, } 2020 \text {, } \\
72,104715\end{array}$ \\
\hline $\mathrm{FeC}_{2} \mathrm{~S}_{4}$ & - & $1 \mathrm{M} \mathrm{KOH}$ & $N_{1}$ foam & - & $100.1 \mathrm{mV} \mathrm{dec}^{-1}$ & $\begin{array}{l}\text { Xiang Wu - Nano Energy, 2020, } \\
72,104715\end{array}$ \\
\hline $\mathrm{FeClO}_{2} \mathrm{~S}_{4}$ & Nanorod arrays & $1 \mathrm{M} \mathrm{KOH}$ & $N_{1}$ foam & $234 \mathrm{mV} @ 50 \mathrm{mAcm}^{-2}$ & - & $\begin{array}{l}\text { Xiang Wu - Nano Energy, 2020, } \\
72,104715\end{array}$ \\
\hline $\mathrm{FeC}_{0_{2}} \mathrm{~S}_{4}$ & Nanosheet arrays & $1 \mathrm{M} \mathrm{KOH}$ & Ti mesh & $340 \mathrm{mV} @ 20 \mathrm{mAcm}^{-2}$ & $104 \mathrm{mV} \mathrm{dec}^{-1}$ & $\begin{array}{l}\text { DaLi - Electrochim. Acta, 2020, } \\
340,135957\end{array}$ \\
\hline $\mathrm{FeCl}_{2} \mathrm{~S}_{4}$ & Nanosheet arrays & $1 \mathrm{M} \mathrm{KOH}$ & $\mathrm{N}_{1}$ foam & $259 \mathrm{mV} @ 20 \mathrm{mAcm}^{-2}$ & $173 \mathrm{mV} \mathrm{dec}^{-1}$ & $\begin{array}{l}\text { Yaqiong Gong - Int. J. Hydrogen } \\
\text { Energy, } 2018,43,17259-17264\end{array}$ \\
\hline
\end{tabular}



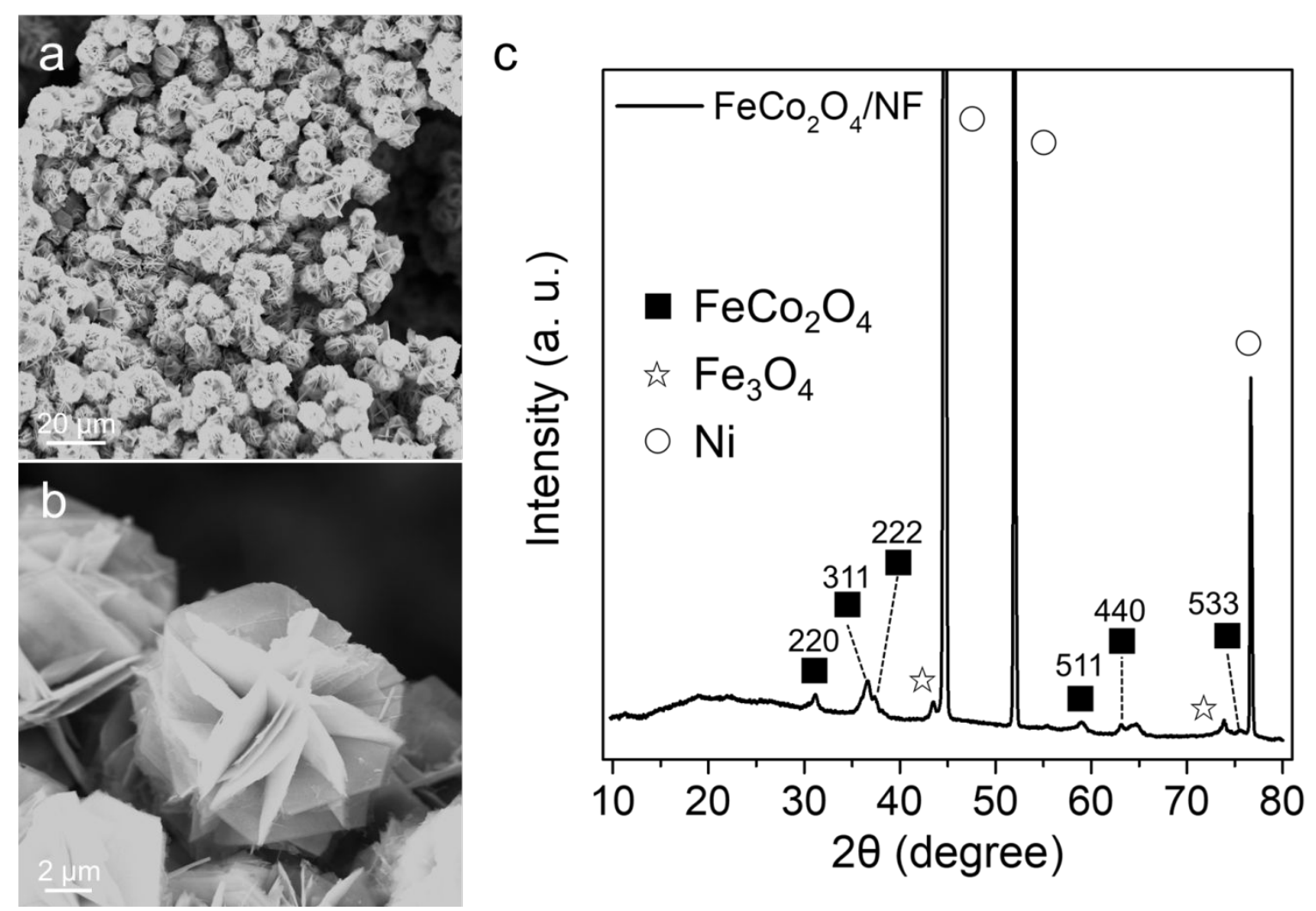

Figure S1. (a, b) SEM images of excavated $\mathrm{FeCo}_{2} \mathrm{O}_{4}$ nanospheres, and (c) its corresponding XRD pattern. 


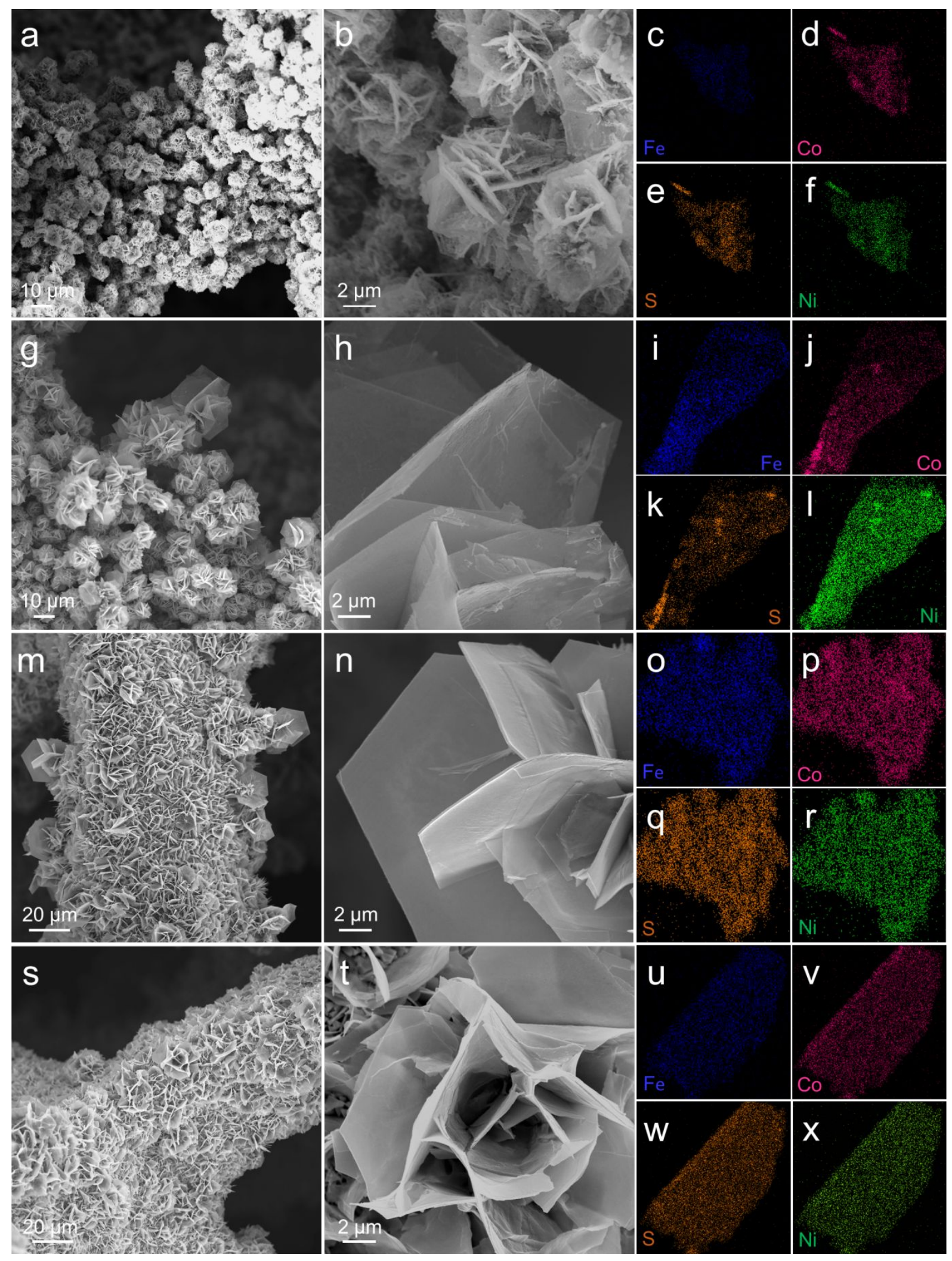

Figure S2. SEM images and STEM-EDS maps of $\mathrm{FeCo}_{2} \mathrm{~S}_{4}$ ENSs made by vulcanization with (a-f) $0.01 \mathrm{M},(\mathrm{g}-1) 0.05 \mathrm{M},(\mathrm{m}-\mathrm{r}) 0.5 \mathrm{M}$, and (s-x) $1 \mathrm{M} \mathrm{Na}_{2} \mathrm{~S}$. 


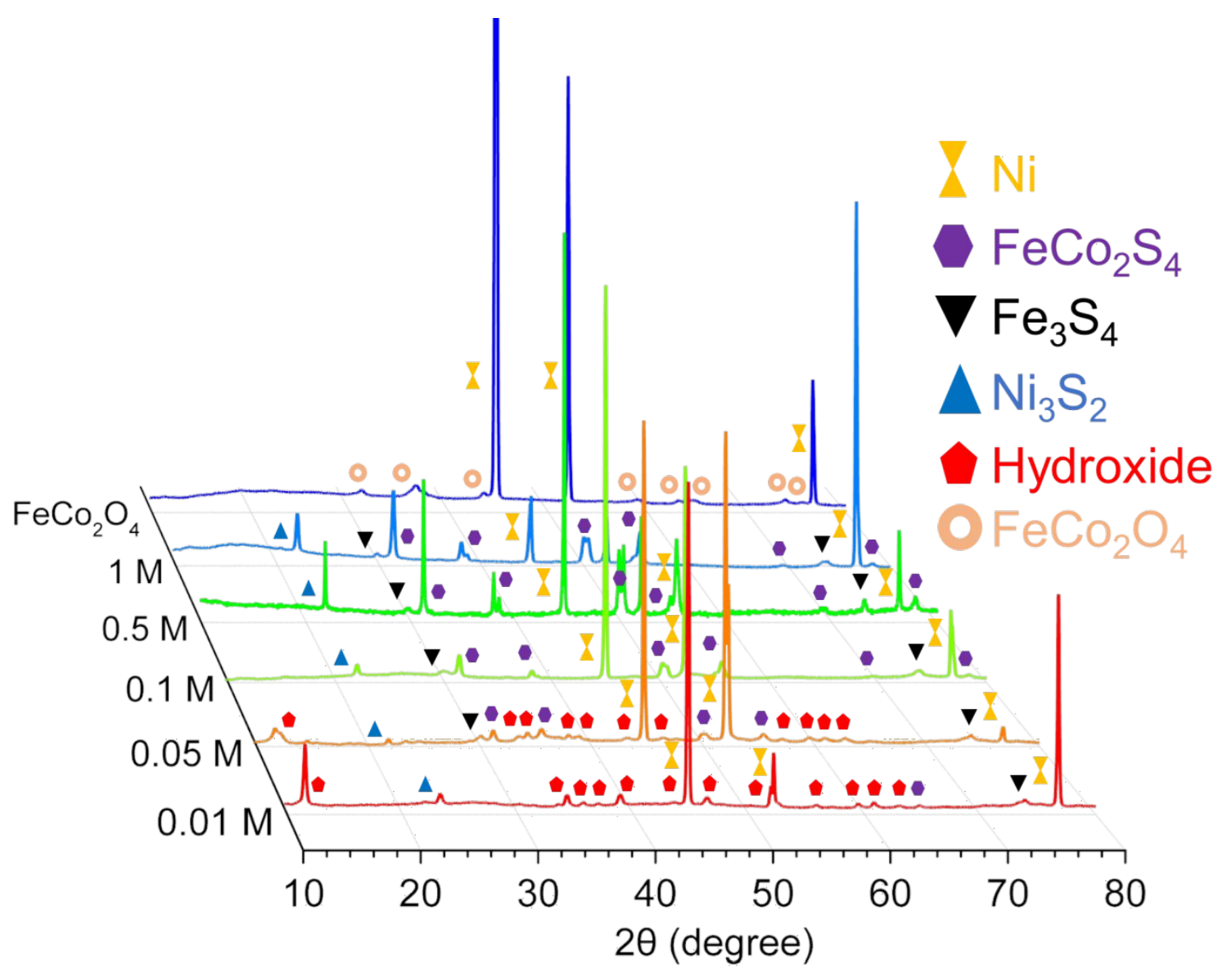

Figure S3. PXRD patterns of Fe-Co HNSs prepared by vulcanization with $0\left(\mathrm{FeCo}_{2} \mathrm{O}_{4}\right), 0.01$, $0.05,0.1,0.5$, and $1 \mathrm{M} \mathrm{Na}_{2} \mathrm{~S}$. 

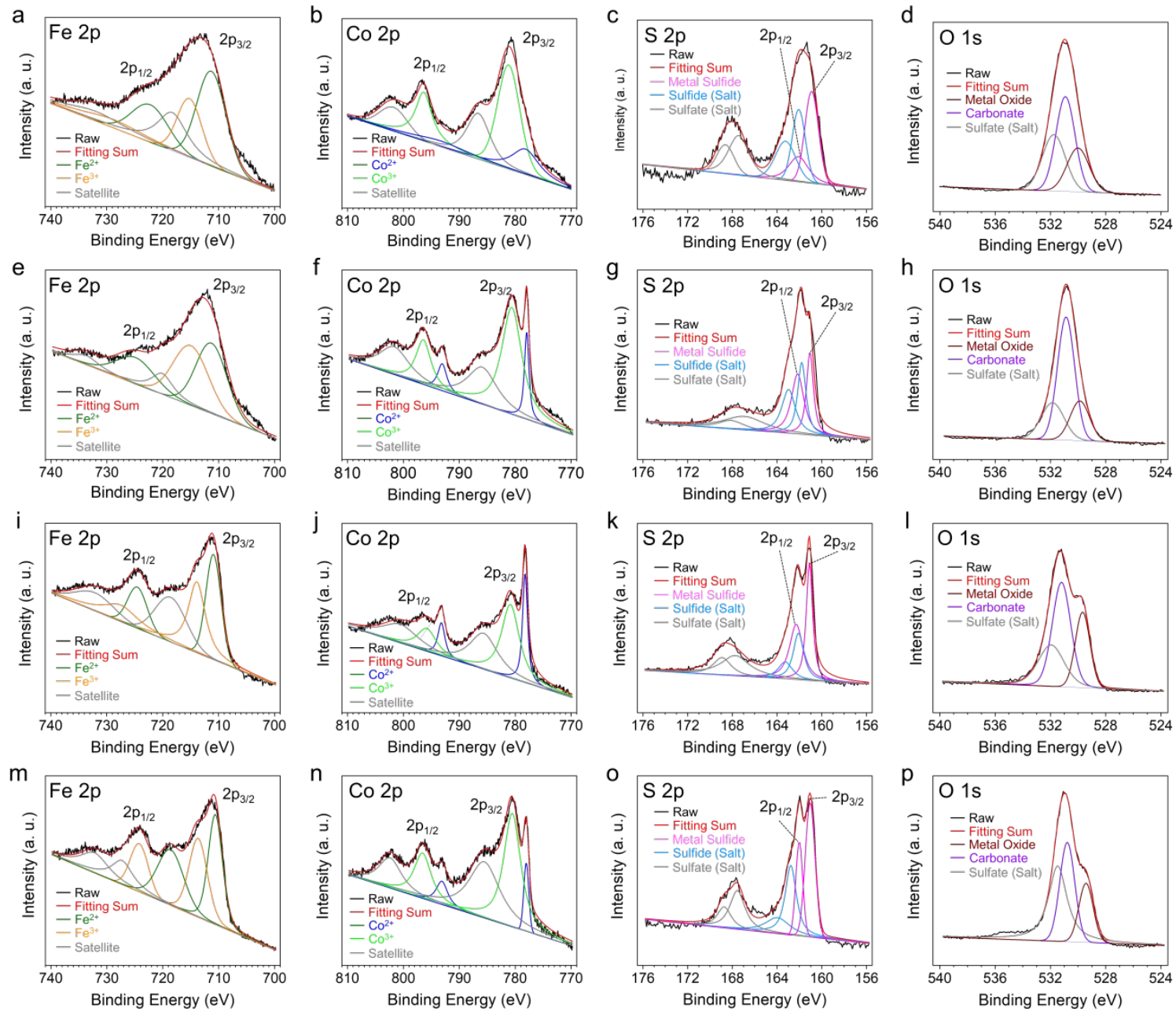

Figure S4. XPS spectra of $\mathrm{Fe}(2 \mathrm{p}), \mathrm{Co}(2 \mathrm{p}), \mathrm{S}(2 \mathrm{p}), \mathrm{O}(1 \mathrm{~s})$, and their fitting curves for

$\mathrm{FeCo}_{2} \mathrm{~S}_{4} / \mathrm{NF}$ made with (a-d) 0.01, (e-h) 0.05, (i-1) 0.5, (m-p) $1 \mathrm{M} \mathrm{Na}_{2} \mathrm{~S}$. 
a

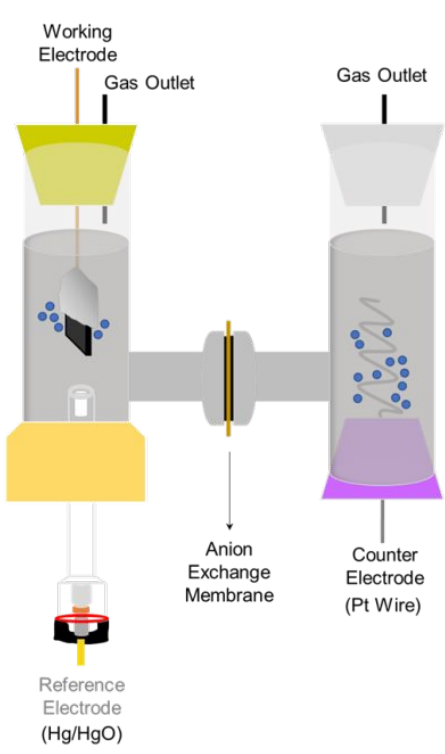

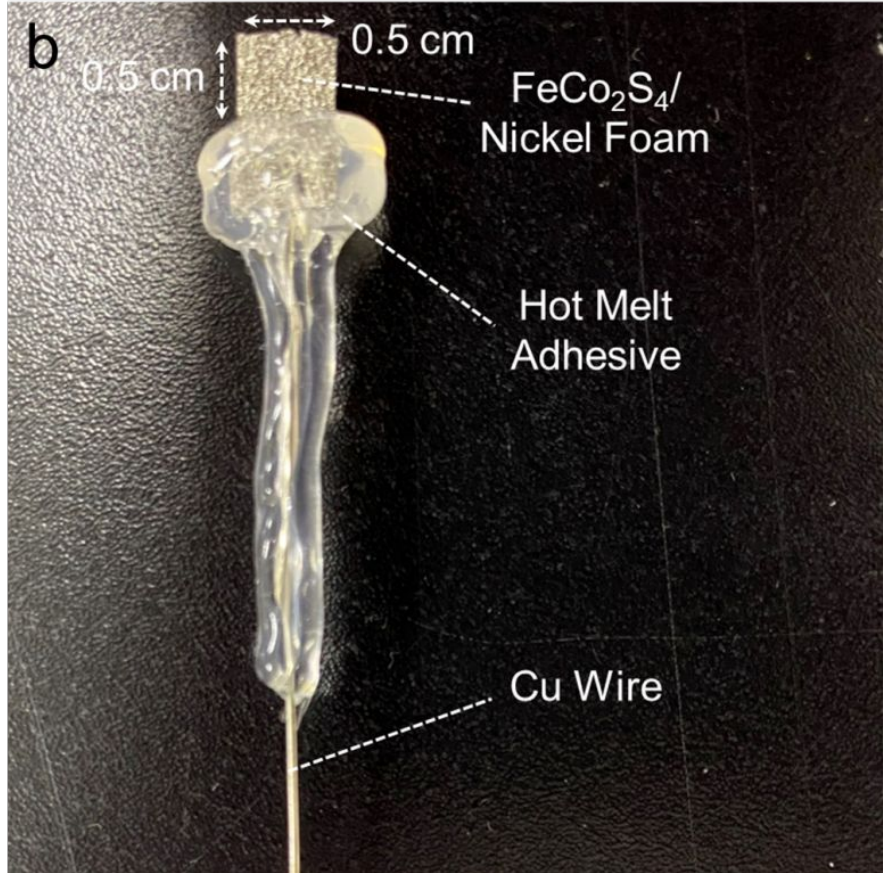

Scheme S1. (a) Schematic illustration for the setup in the H-shaped electrolyzer. (b) Photo of the home-made working electrode. 
a

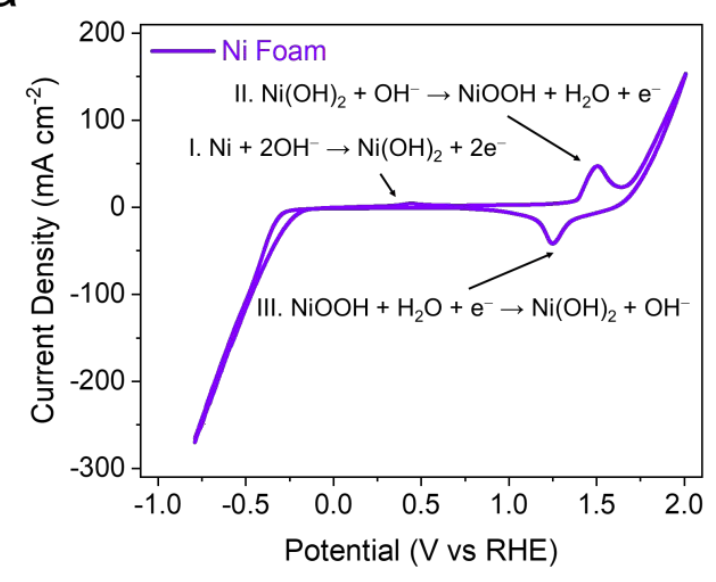

C

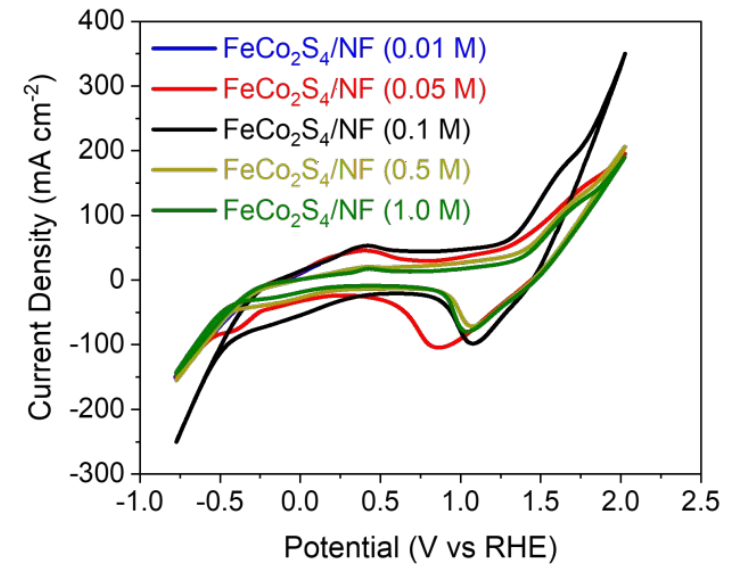

b

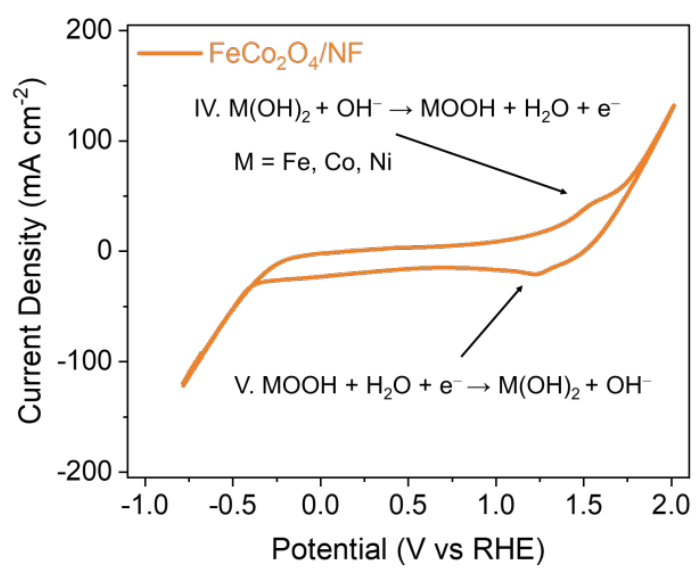

d

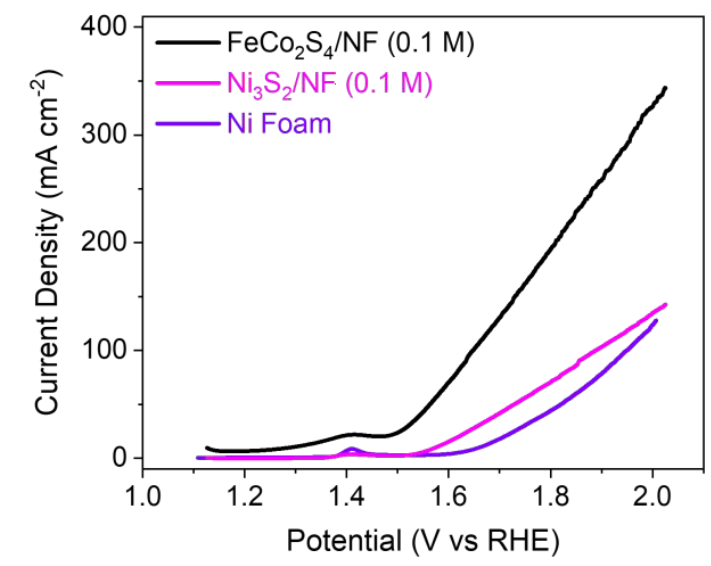

Figure S5. CVs of (a) $\mathrm{Ni}$ foam, (b) $\mathrm{FeCo}_{2} \mathrm{O}_{4} / \mathrm{NF}$, and (c) $\mathrm{FeCo}_{2} \mathrm{~S}_{4} / \mathrm{NF}$ in $1 \mathrm{M} \mathrm{NaOH}$ at a scan rate of $100 \mathrm{mV} / \mathrm{s}$. (d) The LSV curves of $\mathrm{FeCo}_{2} \mathrm{~S}_{4} / \mathrm{NF}(0.1 \mathrm{M})$ and references. 

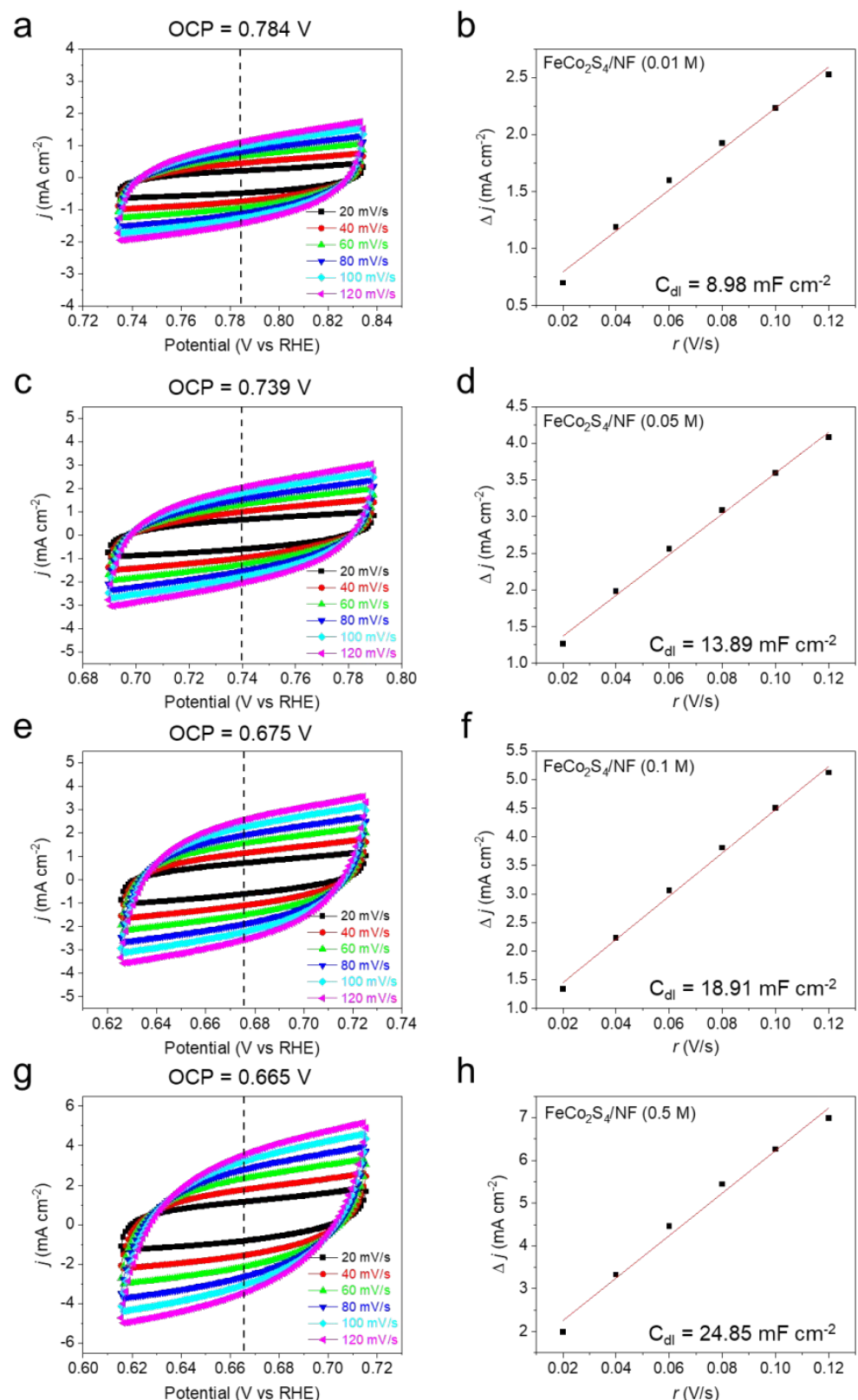

$\mathrm{h}$
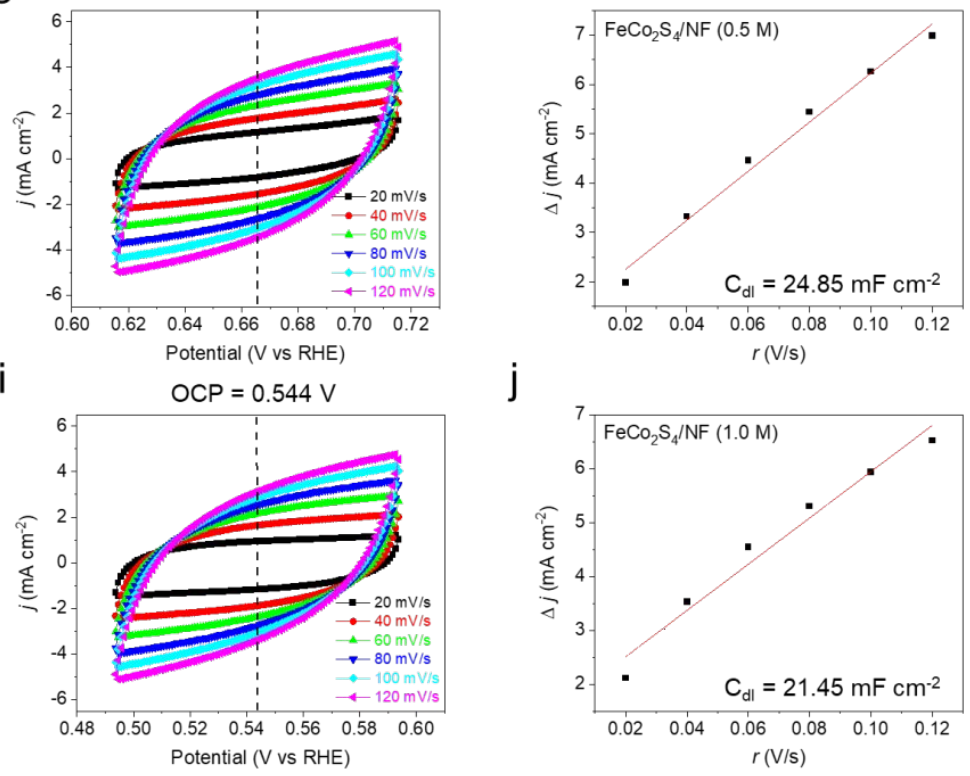

Figure S6. CV curves at scan rates $(r)$ from 20 to $120 \mathrm{mV} / \mathrm{s}$ and their corresponding plots of $\Delta j$ vs $r$ for (a, b) 0.01 M-, (c, d) 0.05 M-, (e, f) 0.1 M-, (g, h) 0.5 M-, and (i,j) $1.0 \mathrm{M}-\mathrm{FeCo}_{2} \mathrm{~S}_{4} / \mathrm{NF}$ electrodes. 
a
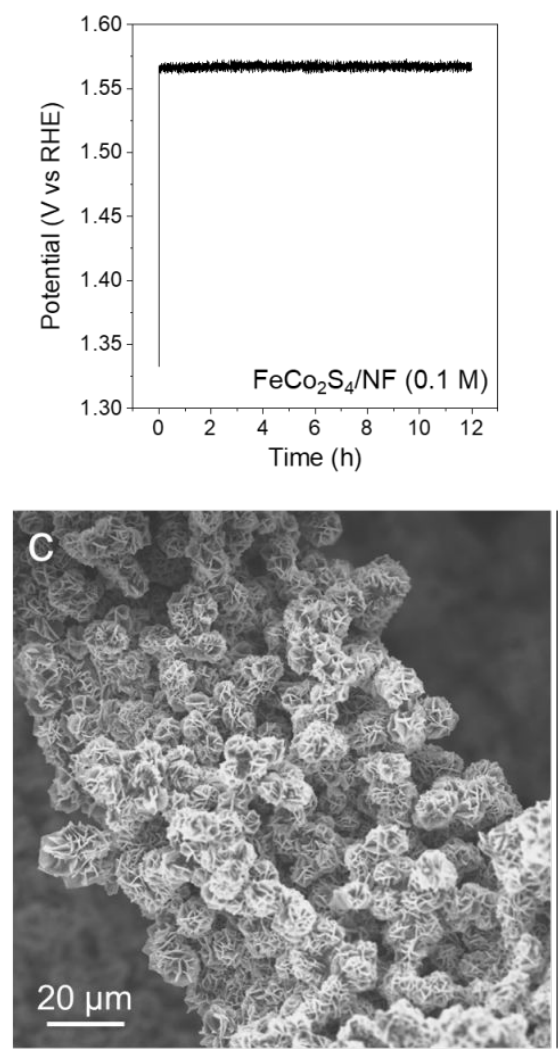

b
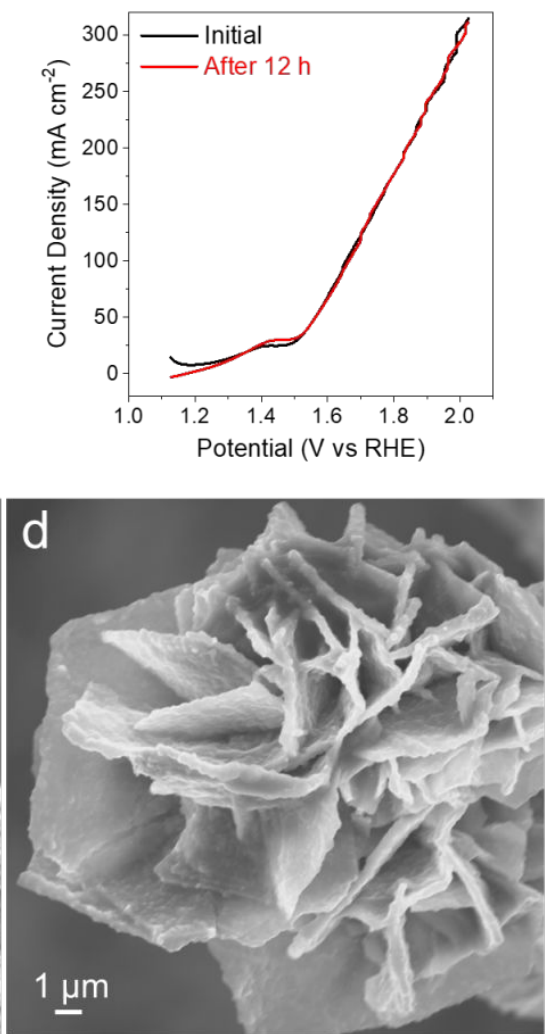

e

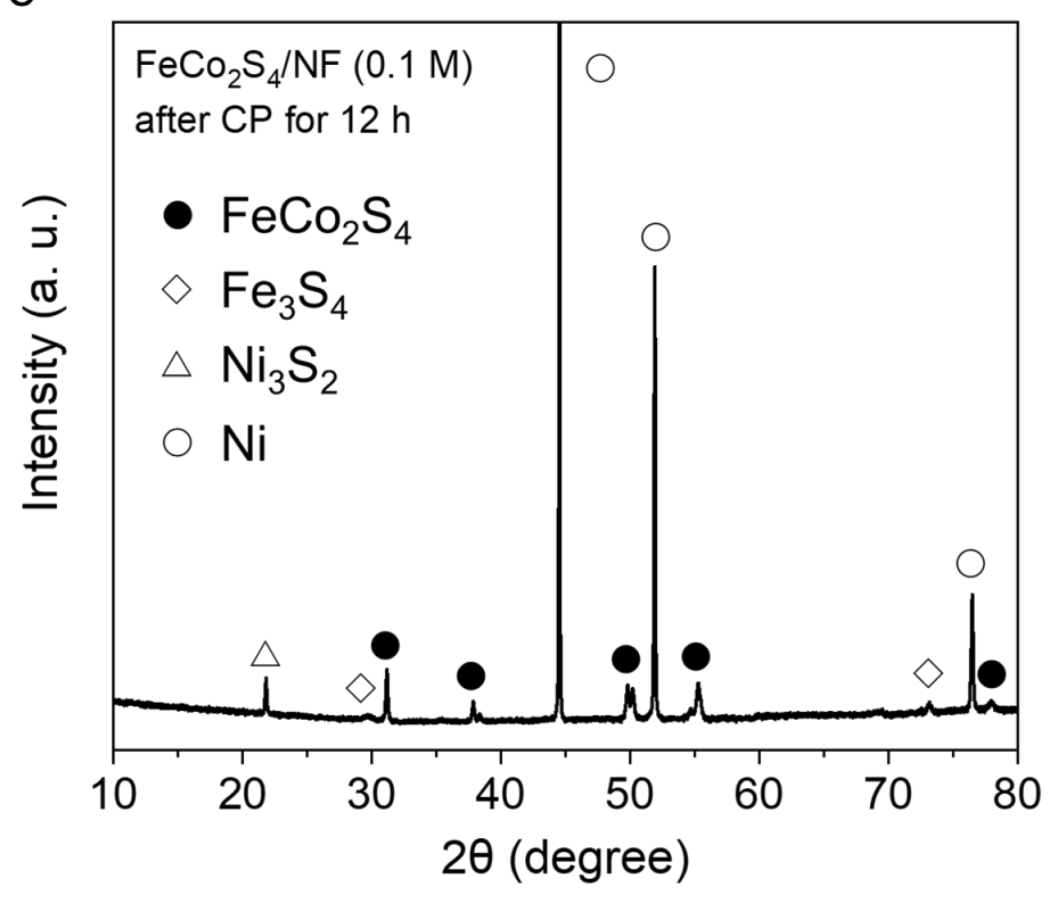

Figure S7. (a) Chronopotentiometry (CP) plot, (b) LSV curve, (c, d) SEM images, and (e) PXRD pattern of $\mathrm{FeCo}_{2} \mathrm{~S}_{4}$ ENSs made with $0.1 \mathrm{M} \mathrm{Na}_{2} \mathrm{~S}$ after 12-hour durability test. The CP plot was obtained by running scan at a static current density of $50 \mathrm{~mA} \mathrm{~cm}{ }^{-2}$ for 12 hours. 
a

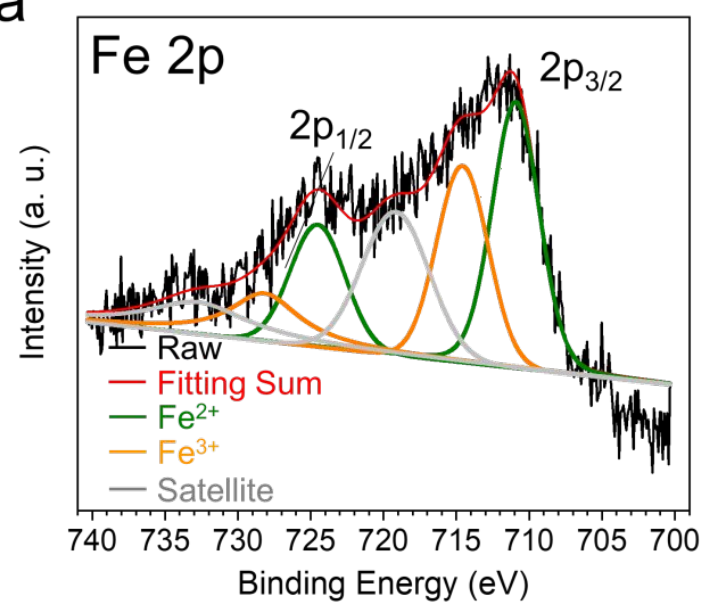

C

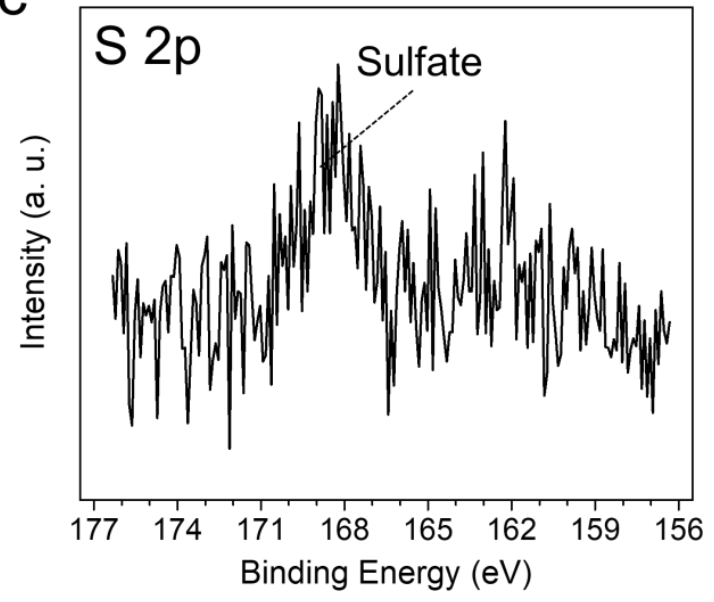

b

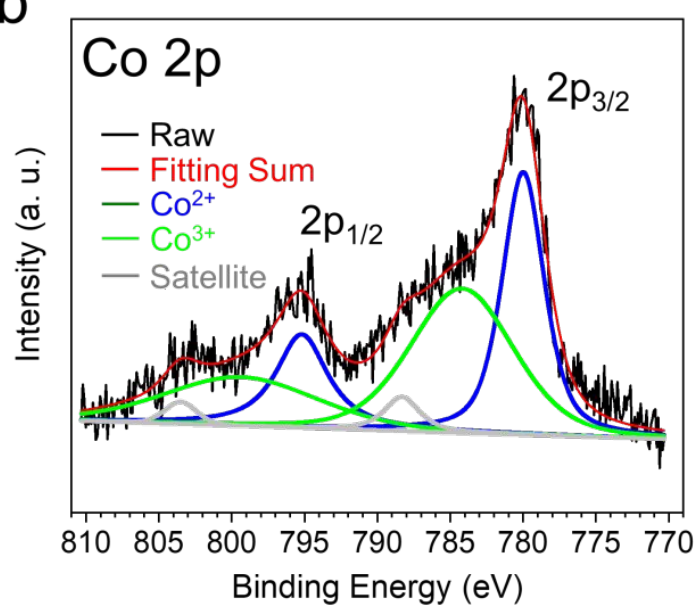

d

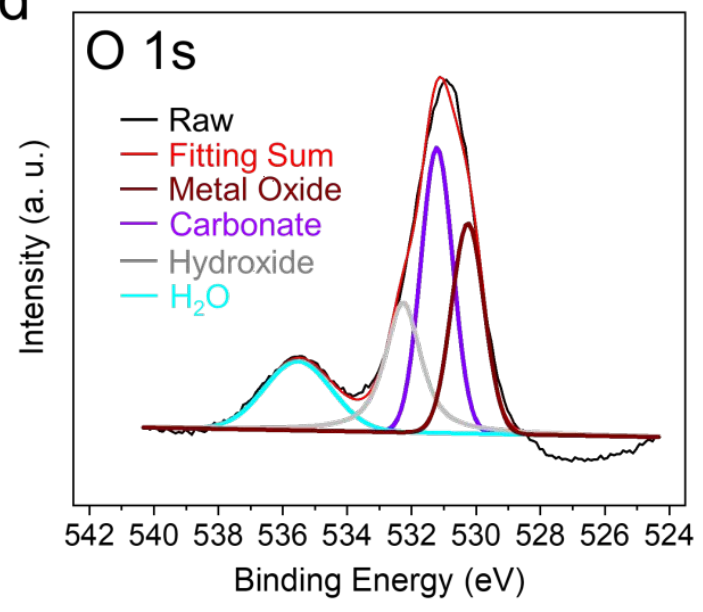

Figure S8. XPS spectra of (a) Fe 2p, (b) Co 2p, (c) S 2p, and (d) O 1s and their fitting curves for $\mathrm{FeCo}_{2} \mathrm{~S}_{4} / \mathrm{NF}\left(0.1 \mathrm{M} \mathrm{Na}_{2} \mathrm{~S}\right)$ after running 12-hour OER. 

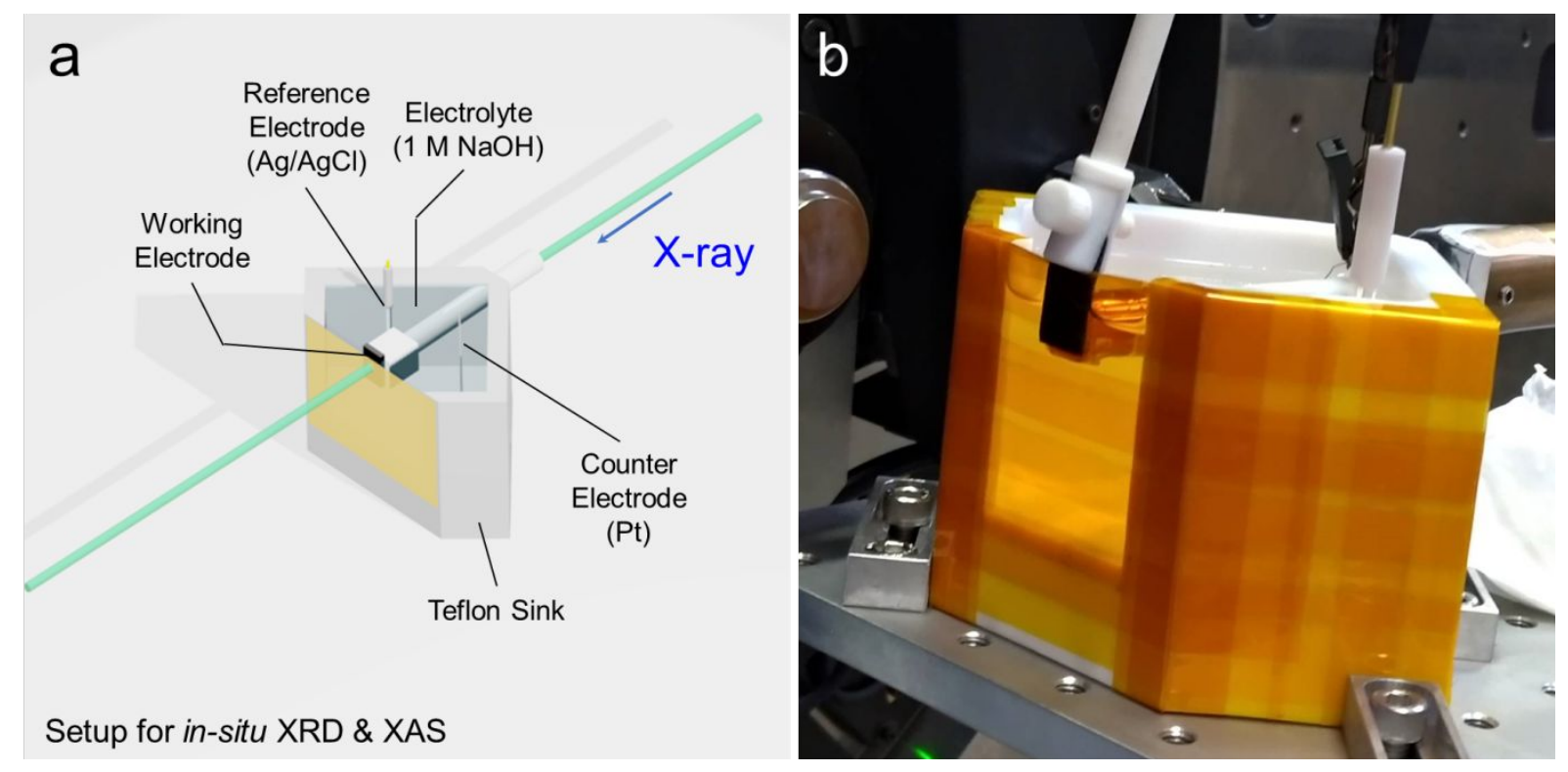

Figure S9. (a) The scheme and (b) photo of the setup for in-situ XRD and XAS measurements during OER at different potentials. 


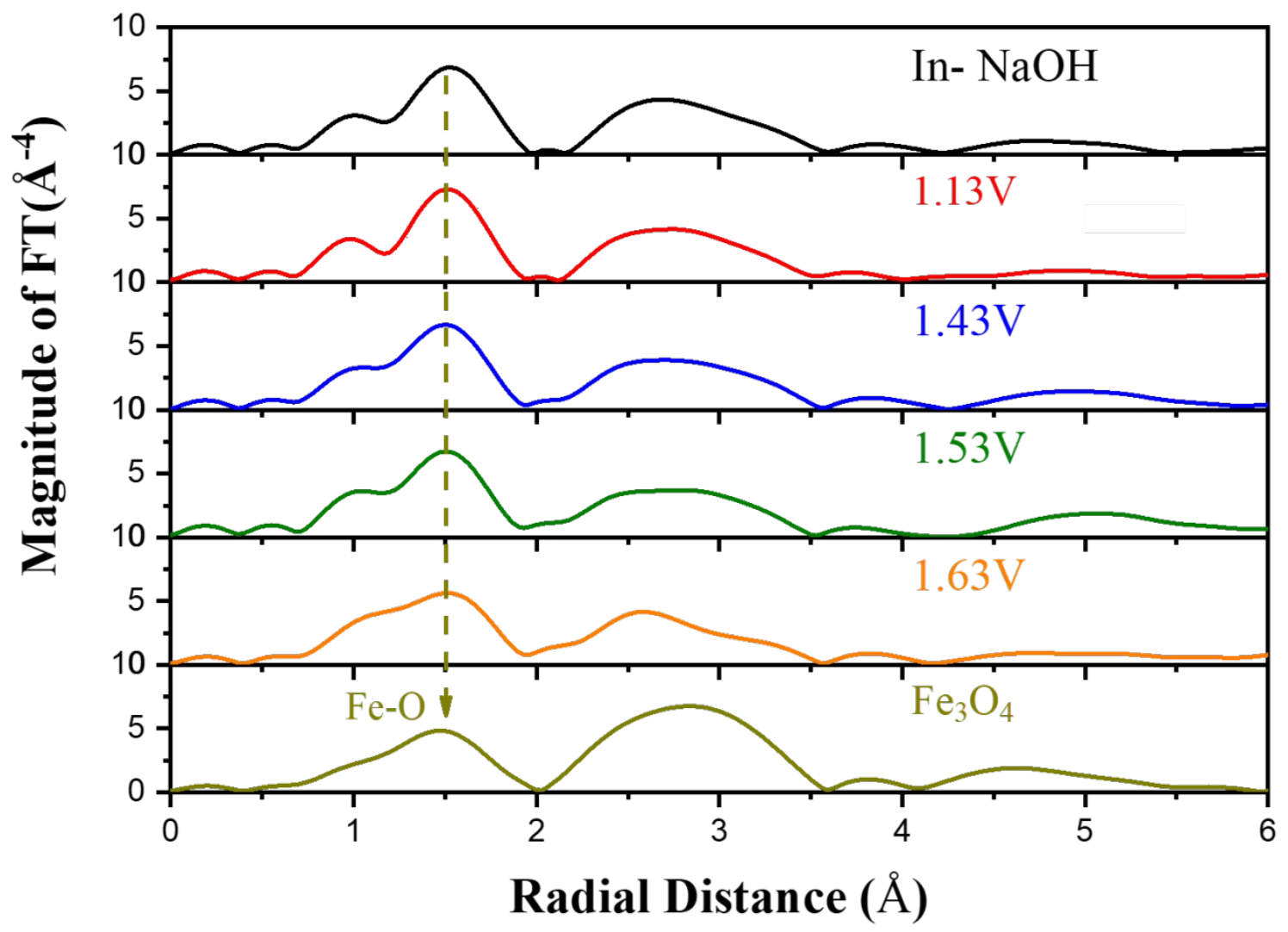

Figure S10. The $k^{3}$-weighted Fourier-transformed EXAFS spectra of Fe K-edge for the $0.1 \mathrm{M}$ -

$\mathrm{FeCo}_{2} \mathrm{~S}_{4} \mathrm{HNS}$ obtained in the electrolyte of $1 \mathrm{M} \mathrm{NaOH}$ at different potentials. 


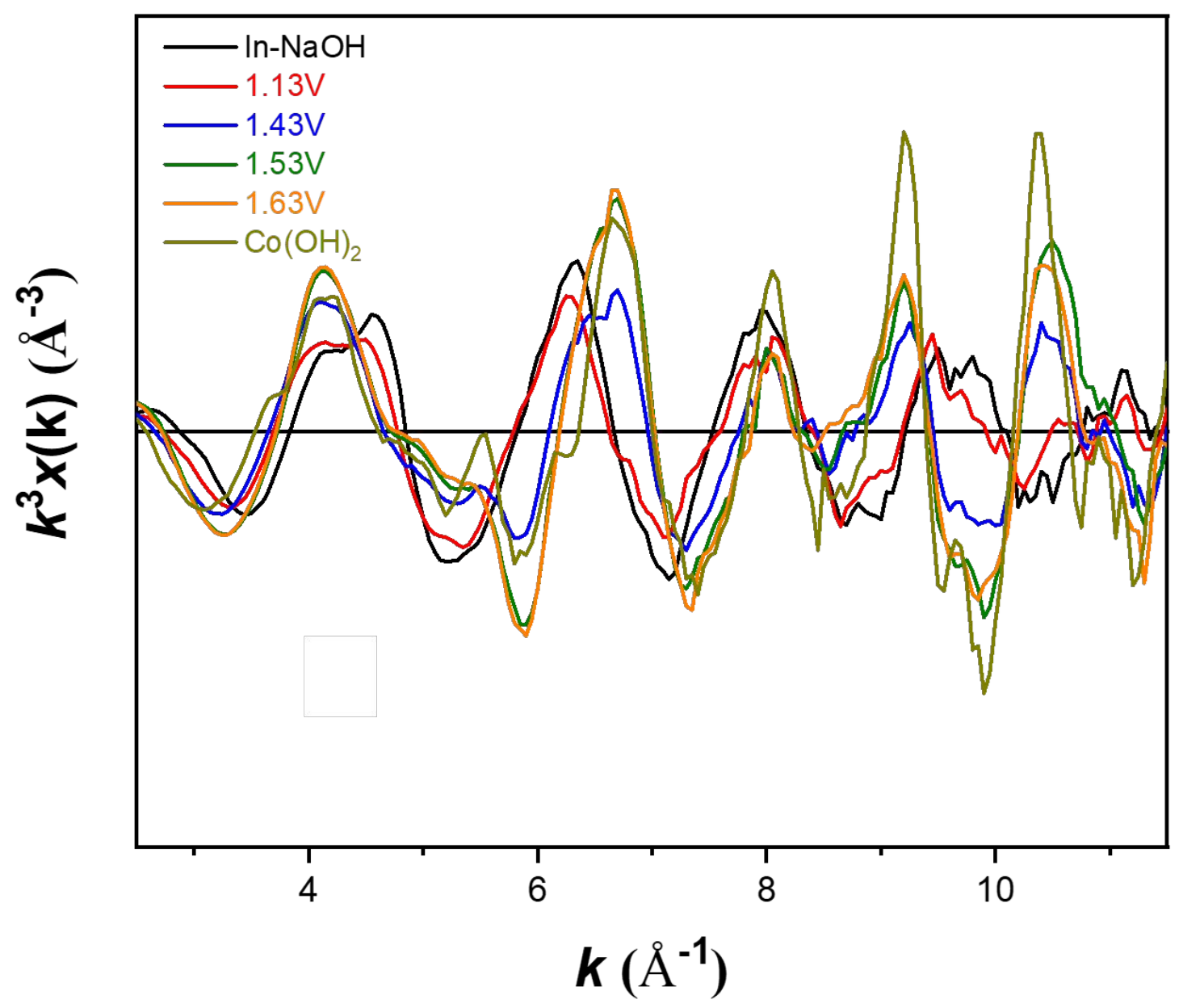

Figure S11. The $k$ space of Co $K$-edge EXAFS spectra for $0.1 \mathrm{M}-\mathrm{FeCo}_{2} \mathrm{~S}_{4} \mathrm{HNS}$ at different potentials. 
Table S2. Structure parameters obtained from in-situ Co $K$-edge EXAFS of the $0.1 \mathrm{M}-\mathrm{FeCo} \mathrm{S}_{4}$ HNS during OER at different potentials.

\begin{tabular}{cccccc}
\hline Sample & Scattering Path & $\mathrm{N}$ & $\mathrm{R}(\AA)$ & $\boldsymbol{\sigma}^{2}\left(\AA^{2}\right)$ & $\mathrm{R}_{\mathrm{f}}$ \\
\hline In-NaOH & Co-S bond & 4.77 & 2.23 & 0.005 & 0.006 \\
$1.13 \mathrm{~V}$ & Co-O bond & 1.10 & 1.89 & 0.005 & 0.004 \\
& Co-S bond & 3.79 & 2.23 & 0.005 & 0.004 \\
$1.43 \mathrm{~V}$ & Co-O bond & 5.50 & 1.91 & 0.006 & 0.040 \\
$1.53 \mathrm{~V}$ & Co-O bond & 5.82 & 1.90 & 0.004 & 0.002 \\
$1.63 \mathrm{~V}$ & Co-O bond & 5.75 & 1.89 & 0.003 & 0.003 \\
$\mathrm{Co}(\mathrm{OH})_{2}$ & Co-O bond & 6 & 1.89 & 0.007 & 0.034
\end{tabular}

Where $\mathrm{N}$ is coordination number, $\mathrm{R}$ is distance between absorber and backscatter atoms, $\sigma^{2}$ is Debye-Waller factor value, $\mathrm{R}_{\mathrm{f}}$ is $\mathrm{R}$-factor characterizing the goodness of fit. $\mathrm{S}_{\mathrm{o}}{ }^{2}$ was fixed to 0.657 as determined from $\mathrm{Co}(\mathrm{OH})_{2}$ fitting. Error bounds (accuracies) characterizing the structural parameters obtained by EXAFS data analysis are estimated to be as follows: $\mathrm{N}, \pm 20 \%$; $\mathrm{R}, \pm 1 \%$; and $\sigma^{2}, \pm 20 \%$. 